Trinity College

Trinity College Digital Repository

Faculty Scholarship

2017

Collective Phenomena Emerging from the Interactions between

Dynamical Processes in Multiplex Networks

Vincenzo Nicosia

Trinity College

Per Sebastian Skardal

Trinity College, persebastian.skardal@trincoll.edu

Alex Arenas

Vito Latora

Follow this and additional works at: https://digitalrepository.trincoll.edu/facpub

Part of the Physical Sciences and Mathematics Commons 


\title{
Collective Phenomena Emerging from the Interactions between Dynamical Processes in Multiplex Networks
}

\author{
Vincenzo Nicosia, ${ }^{1}$ Per Sebastian Skardal, ${ }^{2}$ Alex Arenas, ${ }^{3}$ and Vito Latora ${ }^{1,4}$ \\ ${ }^{1}$ School of Mathematical Sciences, Queen Mary University of London, London E1 4NS, United Kingdom \\ ${ }^{2}$ Department of Mathematics, Trinity College, Hartford, Connecticut 06106, USA \\ ${ }^{3}$ Department d'Enginyeria Informática i Matemátiques, Universitat Rovira i Virgili, 43007 Tarragona, Spain \\ ${ }^{4}$ Dipartimento di Fisica ed Astronomia, Università di Catania and INFN, I-95123 Catania, Italy \\ (Received 26 September 2016; revised manuscript received 17 January 2017; published 31 March 2017)
}

\begin{abstract}
We introduce a framework to intertwine dynamical processes of different nature, each with its own distinct network topology, using a multilayer network approach. As an example of collective phenomena emerging from the interactions of multiple dynamical processes, we study a model where neural dynamics and nutrient transport are bidirectionally coupled in such a way that the allocation of the transport process at one layer depends on the degree of synchronization at the other layer, and vice versa. We show numerically, and we prove analytically, that the multilayer coupling induces a spontaneous explosive synchronization and a heterogeneous distribution of allocations, otherwise not present in the two systems considered separately. Our framework can find application to other cases where two or more dynamical processes such as synchronization, opinion formation, information diffusion, or disease spreading, are interacting with each other.
\end{abstract}

DOI: 10.1103/PhysRevLett.118.138302

Networks are a powerful way to model and study a wide variety of complex phenomena [1,2]. In recent years, the study of collective dynamical processes on complex networks has improved our understanding of many complex systems and shed light on a wide range of physical, biological, and social phenomena including synchronization [3], disease spreading [4], transport [5], and cascades [6]. Of particular interest in these works is the interplay between the structure of the network and its dynamics $[7,8]$. In fact, the topology of a network has an effect on the dynamical processes that take place over the network [9], while some properties of the dynamics can reveal important information on the interaction network [10-12]. Understanding the relations between structure and dynamics can provide a solid foundation for modeling, predicting, and controlling dynamical processes in the real world. However, save for a few notable exceptions [13-16], the majority of the studies so far have considered a single process on a single network, ignoring a very important ingredient: often the components of a complex system interact through two or more dynamics at the same time, and these dynamics usually depend on each other in highly nontrivial ways.

In this Letter we propose a general framework for modeling, through a multiplex network, the coupling of dynamical processes of the same type (e.g., the spreading of two coupled diseases) or of different types (for instance a synchronization dynamics and a diffusion process). Moreover, we demonstrate with a specific example that this coupling mechanism can give rise to the emergence of complex phenomena generated by the interactions between the different dynamical processes.
The natural way to consider $M$ interacting dynamical processes taking place over a complex system is to use a multiplex network with $M$ layers [17-20]. Each layer contains the same number of nodes, $N$, and there exists a one-to-one correspondence between nodes in different layers, but the topology and the very same nature of the connections at each layer may be different. We then assign a different dynamical process to each layer. Considering for simplicity the case $M=2$, we assume that the dynamics of the entire system is governed by the following equations:

$$
\begin{aligned}
\dot{x}_{i} & =F_{\omega_{i}}\left(\mathbf{x}, A^{[1]}\right) \\
\dot{y}_{i} & =G_{\chi_{i}}\left(\mathbf{y}, A^{[2]}\right)
\end{aligned} \quad i=1,2, \ldots, N
$$

where $\mathbf{x}=\left\{x_{1}, x_{2}, \ldots, x_{N}\right\} \in \mathbb{R}^{N}$ and $\mathbf{y}=\left\{y_{1}, y_{2}, \ldots, y_{N}\right\} \in$ $\mathbb{R}^{N}$ denote the states of the two dynamical processes, while the topologies of the two layers are encoded in the adjacency matrices $A^{[1]}=\left\{a_{i j}^{[1]}\right\}$ and $A^{[2]}=\left\{a_{i j}^{[2]}\right\}$, respectively, such that $a_{i j}^{[1]}=1\left(a_{i j}^{[2]}=1\right)$ if a link exists between nodes $i$ and $j$ in the first (second) layer, and $a_{i j}^{[1]}=0$ $\left(a_{i j}^{[2]}=0\right)$ otherwise. The dynamical evolution of the two network processes is ruled, respectively, by the functions $F_{\omega}$ and $G_{\chi}$, which depend on the sets of parameters $\omega$ and $\chi$, so that the state $x_{i}\left(y_{i}\right)$ of node $i$ at the first (second) layer is a function of the state $\mathbf{x}(\mathbf{y})$ and of the topology $A^{[1]}\left(A^{[2]}\right)$ of the first (second) layer. The key ingredient that connects the two dynamical processes is provided by the nature of the correspondence between layers. In fact, the parameter $\omega_{i}$ in function $F_{\omega_{i}}$ at layer 1 is itself a function of time which depends on the dynamical state $y_{i}$ of node $i$ 

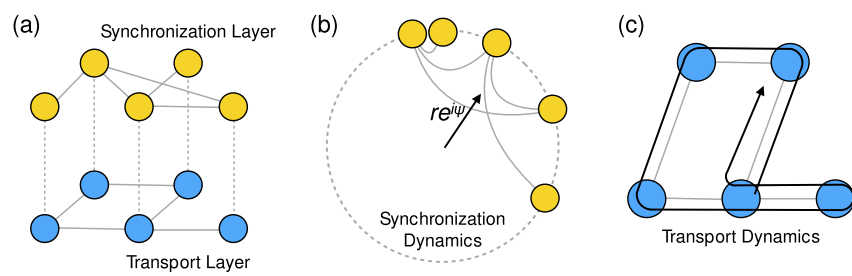

FIG. 1. Intertwined dynamical processes. (a) An example of a two-layer multiplex of $N=5$ nodes with neural synchronization dynamics at layer 1 (top), and transport dynamics at layer 2 (bottom). (b) The neural activity is described by the Kuramoto model in Eq. (3), and the degree of synchronization is measured by the order parameter $r$. (c) The transport dynamics is modeled by biased random walkers moving according to Eq. (4). The two dynamical processes are bidirectionally coupled, as the natural frequencies of the oscillators at layer 1 depend on the distribution of random walkers at layer 2 and, at the same time, the random walkers are biased on the degree of synchronization of the nodes at layer 1, as described in Eqs. (5) and (6).

at layer 2, while the parameter $\chi_{i}$ at layer 2 depends on the state $x_{i}$ of node $i$ at layer 1 . Namely, we have

$$
\begin{aligned}
& \dot{\omega}_{i}=f\left(\omega_{i}, y_{i}\right) \\
& \dot{\chi}_{i}=g\left(\chi_{i}, x_{i}\right)
\end{aligned} \quad i=1,2, \ldots N
$$

where $f$ and $g$ are two assigned functions.

As a specific example of this type of coupling, and of the phenomena that can emerge out of it, we study a toy model of the human brain. Neural systems depend on the combination of several dynamics, including blood flow, oxygen exchange, chemical and electrical interactions among neurons, and remote synchronization of distant regions [21-24]. Our multiplex network approach here wants to mimic the interplay between neural activity and energy transport across brain regions as illustrated in Fig. 1(a). Neural activity at the level of brain regions is modeled by the Kuramoto model [25], such that the state $x_{i}(t) \in[0,2 \pi)$ of node $i$ at layer 1 corresponds to the phase of oscillator $i$ at time $t$, and the first of Eqs. (1) reads

$$
\dot{x}_{i}=\omega_{i}+\lambda \sum_{j=1}^{N} a_{i j}^{[1]} \sin \left(x_{j}-x_{i}\right),
$$

where $\omega_{i}$ corresponds to the natural frequency of the oscillator $i$ and $\lambda$ is the coupling strength. The degree of global synchronization in the neural activity is measured by the Kuramoto order parameter $0 \leq r \leq 1$ defined by the complex number $r e^{i \psi}=(1 / N) \sum_{j=1}^{N} e^{i x_{j}}$ which represents the centroid of all the oscillators on the complex plane. The second dynamical process, namely energy transport at the second layer, is modeled by a continuous-time random walk [26]. Specifically, the state $y_{i}(t) \in[0,1]$ at time $t$ of node $i$ at the transport layer is equal to the fraction of random walkers at node $i$ at time $t$, and the second of Eqs. (1) reads $\dot{y}_{i}=\frac{1}{\tau_{y}} \sum_{j=1}^{N}\left(\pi_{i j}-\delta_{i j}\right) y_{j}=\frac{1}{\tau_{y}} \sum_{j=1}^{N}\left(\frac{a_{j i}^{[2]} \chi_{i}^{\alpha}}{\sum_{l} a_{j l}^{[2]} \chi_{l}^{\alpha}}-\delta_{i j}\right) y_{j}$

where $\pi_{i j}$ is the transition probability from node $j$ to node $i$, $\tau_{y}$ is the time scale of the random walker dynamics, and we have assumed that the random walk is biased on a node property $\chi_{i}$, with a tuneable bias exponent $\alpha$ [27-30]. Notice that for $\alpha>0$ (resp., $\alpha<0$ ) the walkers will preferentially move towards nodes characterized by high (resp., low) values of $\chi$, while for $\alpha=0$ we recover the standard unbiased random walk.

To completely define the model, we have to specify how the neural dynamics and the diffusion of nutrients are coupled; i.e., we need to assign the functions $f$ and $g$ in Eqs. (2), respectively, relating the frequency $\omega_{i}$ of the oscillator $i$ at layer 1 to the available resource $y_{i}$ at layer 2, and the bias property $\chi_{i}$ of the random walkers at layer 2 to the oscillator phase $x_{i}$ at layer 1. First, we assume that the natural frequencies $\omega_{i}, i=1,2, \ldots, N$, evolve dynamically relaxing to values proportional to the fraction of random walkers at node $i$ in the transport layer:

$$
\dot{\omega}_{i}=\frac{1}{\tau_{\omega}}\left[N y_{i}(t)-\omega_{i}\right],
$$

where $\tau_{\omega}$ gives the time scale for the relaxation. This choice is motivated by the fact that firing at a higher frequency usually requires a correspondingly higher amount of energy, in the form of oxygen and nutrients carried by blood [31]. Next, we assume that the quantities $\chi_{i}$ evolve according to

$$
\dot{\chi}_{i}=\frac{1}{\tau_{\chi}}\left(s_{i}^{\mathrm{dyn}}-\chi_{i}\right),
$$

where $s_{i}^{\text {dyn }}$ is the dynamic strength of node $i$, which measures the local degree of synchronization of oscillator $i$ (degree to which $i$ is synchronized with its neighbors) and is defined as $s_{i}^{\mathrm{dyn}}=r_{i} \cos \left(\psi_{i}-x_{i}\right)$ in terms of the local synchronization order parameter $r_{i} e^{i \psi_{i}}=\sum_{j} a_{i j}^{[1]} e^{i x_{j}}$. In this way, $\chi_{i}$ relaxes to the dynamic strength of oscillator $i$ with a time scale $\tau_{\chi}$, and therefore the random walk described by the transition probabilities in Eq. (4) is biased towards (away from) strongly synchronized nodes for positive (negative) values of $\alpha$. This choice is supported by empirical studies confirming the existence of correlations between the electrical activity of a brain area and the hematic inflow in the same area, which is responsible for the transport of energy to the neurons in the form of oxygen molecules. In particular, it has been suggested that the high electrical activity of a brain area is normally followed by an increase in the blood inflow in the same area [32-34].

Summing up, in the model in Eqs. (3)-(6), the firing rate of a given node $i$ depends on the availability of energy at $i$ at the transportation layer, and vice versa the abundance of nutrients at node $i$ depends on the local synchronization of 

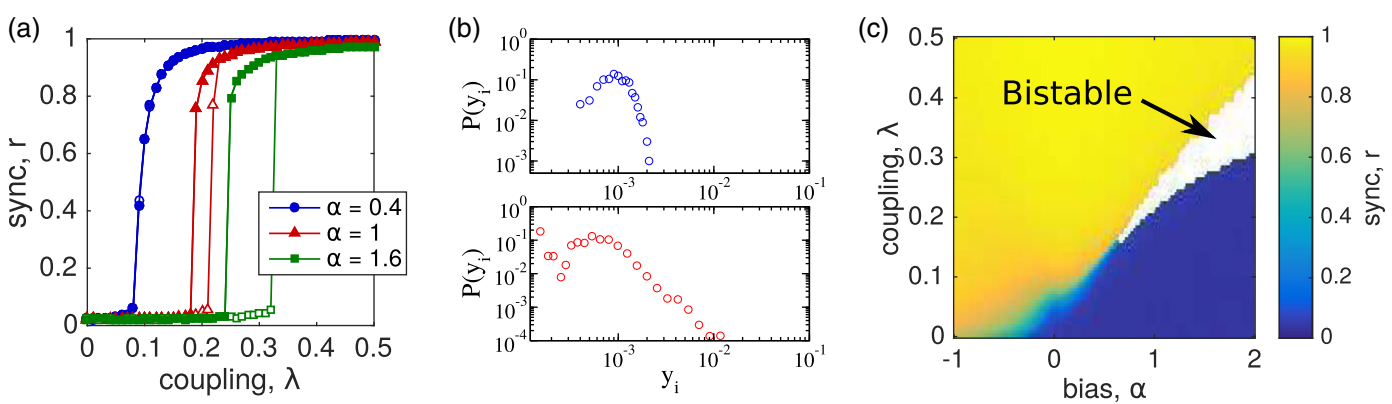

FIG. 2. Spontaneous explosive synchronization induced by the multiplex coupling of the two processes. (a) Level of synchronization $r$ vs $\lambda$ at layer 1 for bias exponents $\alpha=0.4,1.0$, and 1.6 (blue, red, and green, respectively). (b) Distribution $P\left(y_{i}\right)$ of steady-state random walker fractions $y_{i}$ at layer 2 for $\alpha=1.0$, when the oscillators at layer 1 are incoherent $(\lambda=0.1$, top, blue $)$ and synchronized $(\lambda=0.4$ bottom, red). (c) Synchronization phase diagram showing $r$ as a function of coupling $\lambda$ and bias exponent $\alpha$. The bistable region is colored in white. Networks are of size $N=1000$ with $\gamma=3$ and $\left\langle k^{[1]}\right\rangle=\left\langle k^{[2]}\right\rangle=10$.

oscillator $i$ at the neural dynamics layer. Our model has two control parameters, $\lambda$ and $\alpha$, that we can change to tune, respectively, the coupling between oscillators at layer 1 and the strength of the bias in the random walk at layer 2 . To illustrate the effects of intertwining the two dynamical processes, we consider a multiplex network with $N=1000$ nodes whose synchronization layer is a scale-free (SF) graph [35] with degree distribution $P\left(k^{[1]}\right) \propto\left(k^{[1]}\right)^{-\gamma}$ with $\gamma=3$ above a minimum degree $k_{0}^{[1]}$, and whose transport layer is a Erdős-Rényi (ER) random graph [36] with link probability $p$. The average degrees of the two layers are thus given by $\left\langle k^{[1]}\right\rangle=(\gamma-1 / \gamma-2) k_{0}^{[1]}$ and $\left\langle k^{[2]}\right\rangle=p(N-1)$. We choose a SF graph for the synchronization layer given the prevalence of such topologies in real neural systems [37], and we have considered the limits $\tau_{y}, \tau_{\omega}, \tau_{\chi} \rightarrow 0^{+}$corresponding to instantaneous relaxation, meaning the relaxation dynamics of Eqs. (4), (5), and (6) is faster compared to the dynamics of the oscillators. These fast relaxation time scales have been chosen for simplicity, and we note that the phenomena we present here persist for finite values of $\tau_{y}, \tau_{\omega}$, and $\tau_{\chi}$, as we show in the Supplemental Material [38].

We simulated the model on networks with $\left\langle k^{[1]}\right\rangle=$ $\left\langle k^{[2]}\right\rangle=10$, by adiabatically increasing and then decreasing the coupling strength $\lambda$ at fixed values of the bias parameter $\alpha$. In Fig. 2(a) we report the synchronization profiles $r$ vs $\lambda$ for $\alpha=0.4,1.0$, and 1.6 (blue circles, red triangles, and green squares, respectively) at layer 1 . Notice that for $\alpha=$ 0.4 we have the typical continuous phase transition of the Kuramoto model. Conversely, for $\alpha=1.0$ and 1.6 we observe the emergence of a switchlike explosive synchronization [39] and a bistability in the form of a hysteresis loop (in the forward and backward branches of the profiles). In Fig. 2(b) we focus on layer 2, and we plot the distribution $P\left(y_{i}\right)$ of the steady-state random walker occupation probabilities $y_{i}$ for $\alpha=1$, corresponding, respectively, to $\lambda=0.1$ when the system at layer 1 is in an incoherent state (top, blue), and to $\lambda=0.4$ when the system at layer 2 is synchronized (bottom, red). While the values of $y_{i}$ are relatively homogeneous in the incoherent state and span less than a decade, in the synchronized state the distribution is heterogeneous and spanning several decades. Finally, in Fig. 2(c) we explore the $(\alpha, \lambda)$ parameter space in more detail, plotting the value of $r$ at layer 1 as a function of the two control parameters of the model. The bistable region which emerges at $\alpha \approx 0.7$ and widens by increasing $\alpha$ is reported in white. We note that this behavior persists under a wide range of network topologies, provided that the synchronization layer is sufficiently heterogeneous, as shown in the Supplemental Material [38].

Our results indicate that the intertwined nature of diffusion process and synchronization dynamics gives rise to the emergence of phenomena not present if the two dynamics were not coupled. Namely, in the transport layer, we observe a transition from a homogeneous to a heterogeneous distribution of the random walkers throughout the network, according to whether the oscillators at the other layer are incoherent or synchronized. Concurrently, when the random walkers are biased sufficiently strongly towards regions that are more synchronized, the heterogenous distribution of random walkers fosters the emergence of switchlike explosive synchronization [39] in the neural dynamics layer. The resulting phase diagram exhibits three phases (incoherent, bistable, and synchronised) and a tricritical point. It is noticeable that explosive synchronization appears naturally in our model due to the intertwined dynamics of the two processes, and it does not require ad hoc externally imposed correlations between the oscillator frequencies and the topology of the interaction network, as those necessary instead in a single layer network with a single dynamics $[39,40]$.

We now demonstrate that, despite the inherent intricacy of the model, its dynamical behavior can be understood analytically. In particular, we search for conditions such that random walker probabilities and local order parameters are in a stationary state, $y_{i}=y_{i}^{*}$ and $r_{i}=r_{i}^{*}$. A steady-state analysis can then be carried out for both the transport and synchronization dynamics, which we detail in the 

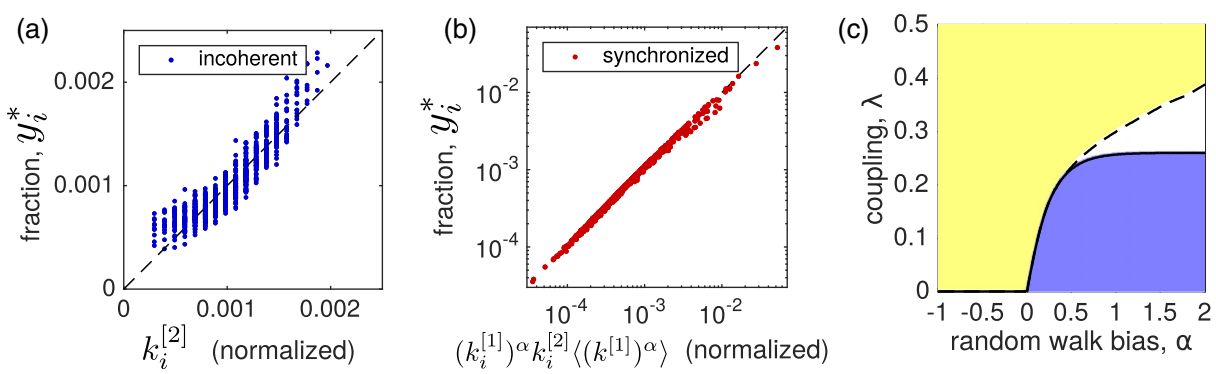

FIG. 3. Analytical approach to explain the observed collective phenomena. Fraction $y_{i}$ of random walkers at node $i$ vs $k_{i}^{[2]}$ for an incoherent state (a), and vs $\left(k_{i}^{[1]}\right)^{\alpha} k_{i}^{[2]}\left\langle\left(k^{[1]}\right)^{\alpha}\right\rangle$ for a synchronized state (b). (c) Analytically obtained synchronization phase diagram showing $r$ as a function of $\lambda$ and $\alpha$. Networks are of size $N=1000$ with $\gamma=3$ and $\left\langle k^{[1]}\right\rangle=\left\langle k^{[2]}\right\rangle=10$ as in the numerical simulations shown in Fig. 2.

Supplemental Material [38]. In particular, we find that the fraction of random walkers $y_{i}^{*}$ depends on whether the synchronization dynamics is incoherent or synchronized, namely,

$$
y_{i}^{*} \propto \begin{cases}k_{i}^{[2]} & \text { if } r \approx 0 \\ \left(k_{i}^{[1]}\right)^{\alpha} k_{i}^{[2]}\left\langle\left(k^{[1]}\right)^{\alpha}\right\rangle & \text { if } r \approx 1 .\end{cases}
$$

Also, the global order parameter $r$ can be written implicitly in terms of the collective frequency $\Omega=\langle\omega\rangle$ and the joint degree-frequency distribution $P(k, \omega)$ :

$r=\frac{1}{\left\langle k^{[1]}\right\rangle} \iint_{|\omega-\Omega| \leq \lambda r k^{[1]}} P\left(k^{[1]}, \omega\right) k^{[1]} \sqrt{1-\left(\frac{\omega-\Omega}{\lambda r k^{[1]}}\right)^{2}} d \omega d k^{[1]}$,

which depends on the topologies of both layers since $\omega_{i}=$ $N y_{i}$ in the steady state. Figure 3 shows that our analytical results are in good agreement with the numerical simulations. In Figs. 3(a) and 3(b) we plot the observed fraction $y_{i}$ of random walkers at the steady state vs the predictions of Eq. (7), respectively, for the incoherent and synchronized state. Dashed black lines are plotted to guide the eye. In Fig. 3(c) we report the synchronization phase diagram obtained from Eq. (8). A comparison with the phase diagram in Fig. 2(c) indicates that our theory is able to accurately reproduce the collective phenomena emerging from the interactions of the two dynamical processes that we have observed in our numerical simulations.

The specific example of intertwined synchronization and transport dynamics studied here shows that interesting collecting behaviors can appear when we couple two dynamical processes taking place on the same set of nodes. Namely, we have found that the distribution of random walkers in the transport network changes from homogeneous to heterogeneous according to whether the synchronization dynamics is incoherent or synchronized, and this result is unexpected since for the topology of the transport network we have on purpose chosen a homogenous graph. At the same time, the heterogeneous distribution of walkers is responsible for the emergence of explosive synchronization, and the appearance of a bistable phase and of a tricritical point in the neural network layer. Importantly, here, explosive synchronization spontaneously emerges from the interactions of the two dynamical processes, without any externally imposed assumptions, necessary instead in networks where the Kuramoto model is not coupled to other dynamical systems $[39,40]$.

The switchlike transition we have found in our model closely mirrors that displayed by the human brain [23], which has the ability to very quickly switch between resting state activity (i.e., the background activity of a brain when no particular conscious task is performed) and complex intellectual or motor tasks [41], and thus requires a fast and flexible mechanism to induce a sudden and massive synchronization. The choice of this specific model was motivated by the important role that synchronization and transport play in a wide range of natural and man-made systems [42-45] and by the various bistabilities empirically observed in physics, biology, and neuroscience $[23,46,47]$. To date, several studies have investigated how a single type of dynamics evolves on a multilayer network [48-51]. However, the framework we have proposed here, based on the use of multiplex networks to mutually couple dynamics of different nature, is very general and versatile. We believe that further studies of other intertwined dynamical processes will uncover other novel phenomena induced by multiplex coupling, and will eventually result in a more thorough understanding of the relation between the structure and the dynamics of multidimensional complex systems.

A. A. and P.S.S. acknowledge support from MULTIPLEX, Grant No. 317532 of the European Commission. V. L. and V. N. acknowledge support from LASAGNE, Grant No. 318132 funded by the European Commission. V. L. acknowledges support from the EPSRC projects GALE, EP/K020633/1, and EP/N013492/1. A. A. acknowledges the Spanish Ministerio de Economiia y Competitividad, Grant No. FIS2015-71582-C2-1, ICREA Academia and the James S. McDonnell Foundation. 
[1] S. H. Strogatz, Exploring complex networks, Nature (London) 410, 268 (2001).

[2] M. E. J. Newman, The structure and function of complex networks, SIAM Rev. 45, 167 (2003).

[3] F. Dörfler, M. Chertkov, and F. Bullo, Synchronization in complex oscillator networks and smart grids, Proc. Natl. Acad. Sci. U.S.A. 110, 2005 (2013).

[4] R. Pastor-Satorras and A. Vespignani, Epidemic Spreading in Scale-Free Networks, Phys. Rev. Lett. 86, 3200 (2001).

[5] J. D. Noh and H. Rieger, Random Walks on Complex Networks, Phys. Rev. Lett. 92, 118701 (2004).

[6] D. B. Larremore, W. L. Shew, and J. R. Restrepo, Predicting Criticality and Dynamic Range in Complex Networks: Effects of Topology, Phys. Rev. Lett. 106, 058101 (2011).

[7] S. Boccaletti, V. Latora, Y. Moreno, M. Chavez, and D. U. Hwang, Complex networks: structure and dynamics, Phys. Rep. 424, 175 (2006).

[8] A. Arenas, A. Díaz-Guilera, J. Kurths, Y. Moreno, and C. Zhou, Synchronization in complex networks, Phys. Rep. 469, 93 (2008).

[9] T. Nishikawa, A. E. Motter, Y.-C Lai, and F. C. Hoppensteadt, Heterogeneity in Oscillator Networks: Are Smaller Worlds Easier to Synchronize?, Phys. Rev. Lett. 91, 014101 (2003).

[10] A. Arenas, A. Díaz-Guilera, and C. J. Pérez-Vicente, Synchronization Reveals Topological Scales in Complex Networks, Phys. Rev. Lett. 96, 114102 (2006).

[11] M. Rosvall and C. T. Bergstrom, Maps of random walks on complex networks reveal community structure, Proc. Natl. Acad. Sci. U.S.A. 105, 1118 (2008).

[12] V. Nicosia, M. De Domenico, and V. Latora, Characteristic exponents of complex networks, Europhys. Lett. 106, 58005 (2014).

[13] S. V. Buldyrev, R. Parshani, G. Paul, H. E. Stanley, and S. Havlin, Catastrophic cascade of failures in interdependent networks, Nature (London) 464, 1025 (2010).

[14] C. Granell, S. Gomez, and A. Arenas, Dynamical Interplay between Awareness and Epidemic Spreading in Multiplex Networks, Phys. Rev. Lett. 111, 128701 (2013).

[15] A. Czaplicka, R. Toral, and M. S. Miguel, Competition of simple and complex adoption on multilayer networks, Phys. Rev. E 94, 062301 (2016).

[16] H. Peng, D. Zhao, X. Liu, and J. Gao, Collective motion in a network of self-propelled agent systems, PLoS One $\mathbf{1 0}$, e0144153 (2015)

[17] M. De Domenico, A. Solé-Ribalta, E. Cozzo, M. Kivelä, Y. Moreno, M. A. Porter, S. Gómez, and A. Arenas, Mathematical Formulation of Multilayer Networks, Phys. Rev. X 3, 041022 (2013).

[18] M. Kivelä, A. Arenas, M. Barthelemy, J. P. Gleeson, Y. Moreno, and M. A. Porter, Multilayer networks, J. Complex Netw. 2, 203 (2014).

[19] S. Boccaletti, G. Bianconi, R. Criado, C. I. del Genio, J. Gómez-Gardeñes, M. Romance, I. Sendiña-Nadal, Z. Wang, and M. Zanin, The structure and dynamics of multilayer networks, Phys. Rep. 544, 1 (2014).

[20] F. Battiston, V. Nicosia, and V. Latora, Structural measures for multiplex networks, Phys. Rev. E 89, 032804 (2014).
[21] F. Varela, J.-P. Lacjaux, E. Rodriguez, and J. Martinerie, The brainweb: Phase synchronization and large-scale integration, Nat. Rev. Neurosci. 2, 229 (2001).

[22] E. Bullmore and O. Sporns, Complex brain networks: Graph theoretical analysis of structural and functional systems, Nat. Rev. Neurosci. 10, 186 (2009).

[23] G. Deco, V. K. Jirsa, and A. R. McIntosh, Resting brains never rest: Computational insights into potential cognitive architectures, Trends Neurosci. 36, 268 (2013).

[24] V. Nicosia, M. Valencia, M. Chavez, A. Díaz-Guilera, and V. Latora, Remote Synchronization Reveals Network Symmetries and Functional Modules, Phys. Rev. Lett. 110, 174102 (2013).

[25] Y. Kuramoto, Chemical Oscillations, Waves, and Turbulence (Springer, New York, 1984).

[26] R. Lambiotte, J.-C. Delvenne, and M. Barahona, Laplacian dynamics and multiscale modular structure in networks, IEEE Trans. Network Science and Engineering 1, 76 (2015).

[27] J. Gómez-Gardeñes and V. Latora, Entropy rate of diffusion processes on complex networks, Phys. Rev. E 78, 065102 (R) (2008).

[28] R. Sinatra, J. Gomez-Gardenes, R. Lambiotte, V. Nicosia, and V. Latora, Maximal-entropy random walks in complex networks with limited information, Phys. Rev. E 83, 030103 (R) (2011).

[29] R. Lambiotte, R. Sinatra, J.-C. Delvenne, T. S. Evans, M. Barahona, and V. Latora, Flowgraphs: Interweaving dynamics and structure, Phys. Rev. E 84, 017102 (2011).

[30] F. Battiston, V. Nicosia, and V. Latora, Efficient exploration of multiplex networks, New J. Phys. 18, 043035 (2016).

[31] Cerebral Blood Flow and Metabolism, edited by L. Edvinsson and D. N. Krause, 2nd ed. (Williams \& Wilkins, Philadelphia, 2002).

[32] D. Malonek, U. Dirnagl, U. Lindauer, K. Yamada, I. Kanno, and A. Grinvald, Vascular imprints of neuronal activity: Relationships between the dynamics of cortical blood flow, oxygenation, and volume changes following sensory stimulation, Proc. Natl. Acad. Sci. U.S.A. 94, 14826 (1997).

[33] S. A. Sheth, M. Nemoto, M. Guiou, M. Walker, N. Pouratian, and A. W. Toga, Linear and nonlinear relationships between neuronal activity, Oxygen metabolism, and hemodynamic responses, Neuron 42, 347 (2004).

[34] E. A. Allen, B. N. Pasley, T. Duong, and R. D. Freeman, Transcranial magnetic stimulation elicits coupled neural and hemodynamic consequences, Science 317, 1918 (2007).

[35] M. Molloy and B. Reed, Critical point for random graphs with a given degree sequence, Random Struct. Algorithms 6, 161 (1995).

[36] P. Erdős and A. Rényi, On the evolution of random graphs, Publ. Math. Inst. Hung. Acad. Sci. 5, 17 (1960).

[37] V. M. Eguíluz, D. R. Chialvo, G. A. Cecchi, M. Baliki, and A. V. Apkarian, Scale-Free Brain Functional Networks, Phys. Rev. Lett. 94, 018102 (2005).

[38] See Supplemental Material at http://link.aps.org/ supplemental/10.1103/PhysRevLett.118.138302 for finite relaxation dynamics, effects of network topology and steady-state analysis.

[39] J. Gómez-Gardeñes, S. Gómez, A. Arenas, and Y. Moreno, Explosive Synchronization Transitions in Scale-Free Networks, Phys. Rev. Lett. 106, 128701 (2011). 
[40] I. Leyva, A. Navas, I. Sendiña-Nadal, J. A. Almendral, J. M. Buldú, M. Zanin, D. Papo, and S. Boccaletti, Explosive transitions to synchronization in networks of phase oscillators, Sci. Rep. 3, 1281 (2013).

[41] M. E. Raichle, A. M. MacLeod, A. Z. Snyder, W. J. Powers, D. A. Gusnard, and G. L. Shulman, A default mode of brain function, Proc. Natl. Acad. Sci. U.S.A. 98, 676 (2001).

[42] J. G. Restrepo, E. Ott, and B. R. Hunt, Onset of synchronization in large networks of coupled oscillators, Phys. Rev. E 71, 036151 (2005).

[43] A. E. Motter, S. A. Myers, M. Anghel, and T. Nishikawa, Spontaneous synchrony in power-grid networks, Nat. Phys. 9, 191 (2013).

[44] P. S. Skardal and A. Arenas, Control of coupled oscillator networks with application to microgrid technologies, Sci. Adv. 1, e1500339 (2015).

[45] D. F. Gleich, PageRank beyond the web, SIAM Rev. 57, 321 (2015).
[46] E. A. Martens, S. Thutupalli, A. Fourrière, and O. Hallatschek, Chimera states in mechanical oscillator networks, Proc. Natl. Acad. Sci. U.S.A. 110, 10563 (2013).

[47] O. Brandman, J. E. Ferrell, R. Li, and T. Meyer, Interlinked fast and slow positive feedback loops drive reliable cell decisions, Science 310, 496 (2005).

[48] F. D. Sahneh and C. Scoglio, Competitive epidemic spreading over arbitrary multilayer networks, Phys. Rev. E 89, 062817 (2014).

[49] K.-M. Lee, C. D. Brummitt, and K.-I. Goh, Threshold cascades with response heterogeneity in multiplex networks, Phys. Rev. E 90, 062816 (2014).

[50] M. Pósfai, J. Gao, S. P. Cornelius, A.-L. Barabási, and R. M. D'Souza, Controllability of multiplex, multi-time-scale networks, Phys. Rev. E 94, 032316 (2016).

[51] R. Burkholz, M. V. Leduc, A. Garas, and F. Schweitzer, Systemic risk in multiplex networks with asymmetric coupling and threshold feedback, Physica D (Amsterdam) 323-324, 64 (2016). 